\title{
Neues vom heutigen Deutsch
}

Empirisch - methodisch - theoretisch

Herausgegeben von

Ludwig M. Eichinger und Albrecht Plewnia 
ISBN 978-3-11-062065-8

e-ISBN (PDF) 978-3-11-062259-1

e-ISBN (EPUB) 978-3-11-062075-7

ISSN 0537-7900

\section{Library of Congress Control Number: 2018967886}

\section{Bibliografische Information der Deutschen Nationalbibliothek}

Die Deutsche Nationalbibliothek verzeichnet diese Publikation in der Deutschen

Nationalbibliografie; detaillierte bibliografische Daten sind im Internet über http://dnb.dnb.de abrufbar.

(C) 2019 Walter de Gruyter GmbH, Berlin/Boston

Satz und Layout: Joachim Hohwieler, Annett Patzschewitz

Druck und Bindung: $\mathrm{CPI}$ books $\mathrm{GmbH}$, Leck

www.degruyter.com 


\title{
Vilmos Ágel (Kassel) \\ Grammatische Textanalyse (GTA) - eine deszendente Syntax des Deutschen
}

\begin{abstract}
Der Blick auf die Syntax und generell auf die Grammatik ist traditionell aszendent, ,von unten nach oben' gerichtet: Einer Wortgrammatik folgt eine Satzgrammatik und dieser eventuell eine Textgrammatik. Doch wir schreiben und sprechen weder in Wörtern noch in Sätzen, sondern wir produzieren Texte und Gespräche. Deshalb müsste auch der diametral entgegengesetzte Blick, der zu einer deszendenten Grammatik führt, möglich sein. Eine solche Grammatik liegt mit der Grammatischen Textanalyse (= GTA), einer funktionalen Syntax des Gegenwartsdeutschen vor, die das grammatische System ,von oben nach unten ${ }^{\text {‘ }}$ - von der Text- (Textglieder) über die Satz- (Satzglieder) zur Wortgruppenebene (Wortgruppenglieder) - modelliert. Im Beitrag werden Grundlagen und Leitbegriffe der GTA vorgestellt und an ausgewählten Phänomenen exemplifiziert.
\end{abstract}

\section{Was von der GTA zu erwarten ist}

Im vorliegenden Beitrag soll es um die kurze Vorstellung eines grammatischen Modells gehen, das unter dem Titel „Grammatische Textanalyse. Textglieder, Satzglieder, Wortgruppenglieder“ (= GTA 2017) erschienen ist. ${ }^{1}$

Der Blick auf die Syntax und generell auf die Grammatik ist traditionell aszendent, ,von unten nach oben' gerichtet: Einer Wortgrammatik folgt eine Satzgrammatik und dieser eventuell eine Textgrammatik. Doch bekanntlich schreiben und sprechen wir weder in Wörtern noch in Sätzen, sondern wir produzieren Texte (inkl. „textes oraux“; Adam 2005, S. 122). Deshalb müsste auch der diametral entgegengesetzte Blick, der zu einer deszendenten Grammatik führt, möglich sein. Eine solche Grammatik liegt mit der GTA, einer funktionalen Syntax des Gegenwartsdeutschen vor, die das grammatische System ,von oben nach unten` - von

\footnotetext{
1 Die Vorstellung kann angesichts des Umfangs des Buches - 789 Seiten ohne Apparat - tatsächlich nur sehr kurz (und verkürzt) erfolgen. Dabei werden zahlreiche Formulierungen ohne Selbstzitat aus der GTA übernommen. Sonst würde der vorliegende Beitrag überwiegend aus Selbstreferenzen bestehen. Generell gilt, dass alle Theoriebausteine, die in dem vorliegenden Beitrag vorgestellt werden, in der GTA wesentlich ausführlicher beschrieben und da auch begründet werden.
} 
der Text- (Textglieder) über die Satz- (Satzglieder) zur Wortgruppenebene (Wortgruppenglieder) - modelliert. Die GTA ist also keine Textgrammatik, sondern eine ,auf den Kopf gestellte‘ Syntax, die auf der Textebene ansetzt.

Aszendenz und Deszendenz, die nicht mit Projektionismus vs. Konstruktionismus (siehe Jacobs 2008, 2009; Welke 2011) gleichzusetzen sind, stellen zumindest idealiter komplementäre Forschungslogiken dar: Baut man eine Brücke von beiden Seiten des Ufers her, erwartet (oder hofft) man, dass sich die Brückenhälften in der Mitte treffen und auch exakt passen. Doch bei Aszendenz vs. Deszendenz sind die ,beiden Seiten des Ufers‘ schwer zu vergleichen: ein einfaches Wort vs. ein beliebig komplexer Text.

Die Chance, dass sich beliebig komplexe Texte ausgehend von einfachen Wörtern exhaustiv und widerspruchsfrei nach oben hin ableiten lassen, ist gering. Denn nicht nur Satzglieder sind Glieder von Sätzen, auch Sätze sind Glieder von Texten. Deshalb ist die Rekonstruktion der - grammatischen (und nicht der orthografischen) - Satzgrenze keine bloße ,innere Angelegenheit“ des Satzes, sondern sie hängt auch von der Modellierung der Textglieder ab.

Umgekehrt zwingt der Ausgang vom Text den Grammatiker dazu, gemäß dem Matroschka-Prinzip zu agieren: Er hat alle Makroglieder (Textglieder) zu beschreiben, dann alle Mesoglieder von allen Sätzen (Satzglieder) und schließlich alle Mikroglieder von allen Satzgliedern (Wortgruppenglieder und andere Mikroglieder).

Der ,Weg nach unten' betrifft also drei Ebenen und drei Analyseeinheiten, denen jeweils ein grammatischer Wert, eine ,Matroschka', zugeordnet ist:

Tab. 1: Ebenen, Analyseeinheiten und Werte

\begin{tabular}{lll}
\hline Ebene & Analyseeinheit & grammatischer Wert \\
\hline Makroebene & Text & Textglied \\
Mesoebene & Satz & Satzglied \\
Mikroebene & Wortgruppe & Wortgruppenglied \\
\hline
\end{tabular}

Auf dem ,Weg nach unten' darf dabei keine Vereinfachung vorgenommen werden und keine Struktur unberücksichtigt bleiben.

Der Ausgang vom Text führt notwendigerweise $\mathrm{zu}$ einer funktionalen Syntax in dem Sinne, dass im Zentrum des grammatischen Modells grammatische Werte stehen, die terminologisch, wenn möglich, durch das Grundwort ...glied eines Kompositums gekennzeichnet sind. Diese stellen das Resultat der Anwendung einer bestimmten grammatischen Funktion auf eine bestimmte grammatische Form dar (siehe Kap. 2). 
Was aber ist von einer Grammatik im Allgemeinen und was von der GTA im Besonderen zu erwarten? Einen Leitfaden hierzu bietet die IDS-Grammatik (1997, S. $3 \mathrm{f}$.$) :$

(a) Grammatiken sollen auf maximale Extension des Gegenstandsbereichs zielen, sie sollen eine Einzelsprache deskriptiv vollständig in ihrer mündlichen und schriftlichen Ausprägung erfassen, möglicherweise noch in ihrer Aufgliederung in historische, regionale oder soziale Varietäten.

(b) Grammatiken sollen theoretisch fundiert und homogen sein, sie sollen größtmögliche wissenschaftliche Aktualität bieten, auch in der Untersuchung der einzelnen Phänomene.

(c) Grammatiken sollen die Teilbereiche des sprachlichen Systems wohlproportioniert behandeln und keine theoretisch nicht legitimierbaren Gewichtungen haben.

(d) Grammatiken sollen universellen Fragestellungen zugänglich sein, d.h. die Besonderheiten der Sprache, wie sie für Typologie- und Universalienforschung relevant sind, hervortreten lassen und ein Begriffsnetz verwenden, das auch für andere Sprachen verwendet wird oder verwendbar ist.

(e) Grammatiken sollen nicht normativ sein, sondern die Sprachwirklichkeit zum Gegenstand machen.

(f) Grammatiken sollen Handbücher zur Problemlösung sein, d.h. eher Resultate als Lösungswege, Theorien- oder Alternativendiskussion präsentieren.

Was ist nun - im Lichte dieser Punkte - von der GTA zu erwarten?

Gemäß dem „Vollständigkeitsparadox“ der IDS-Grammatik (1997, S. 4) schließen sich „deskriptive Vollständigkeit“ (= (a)) und ,theoretische Fundierung“ (= (b)) derzeit aus: „Vollständigkeit geht auf Kosten theoretischer Konsistenz und umgekehrt“(ebd.).

Da die GTA eine wissenschaftliche Grammatik ist und da wissenschaftliche Grammatiken „eher den Anspruch auf Vollständigkeit als den auf theoretische Fundierung abschwächen“ (ebd.), sollen die nachfolgenden Kapitel vor allem (b), d.h. den theoretischen Grundlagen und Leitbegriffen der GTA bzw. deren Exemplifizierung an ausgewählten Phänomenen der deutschen Gegenwartsgrammatik, gewidmet werden.

Was deskriptive Vollständigkeit anbelangt, sie ist einerseits variationsbezogen, andererseits phänomenbezogen zu verstehen.

Das Streben nach variationsbezogener deskriptiver Vollständigkeit bedeutet im Hinblick auf die Standardsprache (siehe Schmidt/Herrgen 2011, S. 59 ff.), dass in der GTA auch syntaktische Phänomene berücksichtigt wurden, die spezifisch für Österreich oder die Schweiz sind. Im Hinblick auf Schriftlichkeit/Mündlichkeit liegt der Fokus zwar auf der Schriftlichkeit, doch auch Phänomene der (echten oder literarisch simulierten) mündlichen Alltagssprache wurden herangezogen.

Das Streben nach phänomenbezogener deskriptiver Vollständigkeit bedeutet das Streben nach Exhaustivität sowohl auf der Text- als auch auf der Satzebene. 
Da diese Punkte eng mit (b) verzahnt sind, können sie in die Vorstellung der theoretischen Grundlagen integriert werden (siehe Kap. 3 und 5).

Aus (a) und (b) ergibt sich somit folgender Aufbau:

- Funktion-Argument-Wert-Formel (Kap. 2),

- Exhaustivität auf Textebene (Kap. 3),

- Rekursivität, Recycling, Deszendenz (Kap. 4),

- Restlosigkeit der Satzanalyse (Kap. 5),

- Grammatische Grundstruktur als semantischer Differenzwert (Kap. 6) und

- Statik und Dynamik (Kap. 7).

Die Erwartungen (c) bis (f) können hier nur kurz kommentiert werden:

- ad (c): Die Teilbereiche des syntaktischen Systems in der GTA sind die Makroebene (Textebene), die Mesoebene (Satzebene) und die Mikroebene (Wortgruppenebene). Entsprechend der syntaktischen Tradition und dem Erforschungsgrad der Ebenen entfallen dabei ca. 60\% des Umfangs auf die Mesoebene. Die Makroebene umfasst ca. 25\%, die Mikroebene ca. 15\%.

- ad (d): Verbindungen zur Typologie- und Universalienforschung wurden in der GTA systematisch angestrebt. Dies zeigt sich besonders deutlich bei folgenden Themen: Reflexivität und Medialität, Kopula+Prädikativ als Prädikat, Subjektlosigkeit und Empfindungsprädikate, Transfer- und Benefaktiv-Alternation, Pertinenzdativ und externe Possession, Mikro- und Makrosatzglieder, Passive.

- ad (e): Die GTA ist nicht normativ, sondern macht „die Sprachwirklichkeit zum Gegenstand“. Dies geht so weit, dass auch Texte von Autoren, deren Sprache mitunter als ,schlechtes Deutsch' abgelehnt wird, berücksichtigt werden (z.B. Wolf Haas). Wie sich Ungrammatikalität und sogenannte Performanzfehler abgrenzen lassen, wurde am Beispiel der Apokoinu-Konstruktionen gezeigt (GTA 2017, S. 153 ff.).

- ad (f): Da „Grammatiken eher benutzt als gelesen werden“ (IDS-Grammatik 1997, S. 7), plädiert die IDS-Grammatik tendenziell für eine Resultatsgrammatik und nicht für eine Problemgrammatik (zur Unterscheidung siehe Helbig 1992, S. 142 f.). In der GTA wurden allerdings „strukturelle Maßnahmen“ ergriffen, um die Lektüre den anvisierten Benutzergruppen anzupassen (siehe GTA 2017, S. XIV-XVI). Eine dieser Maßnahmen ist der zunehmende Schwierigkeitsgrad innerhalb der einzelnen Hauptkapitel. Dies impliziert u.a., dass tendenziell resultatsgrammatische Kapitel zunehmend in problemgrammatische übergehen. 


\section{Funktion-Argument-Wert-Formel}

Die Grundidee des Konzepts der Grammatischen Textanalyse ist, dass sich die Architektur der Grammatik in Analogie zu einer einfachen logischen Formel beschreiben lässt (siehe Allwood/Andersson/Dahl 1973, S. 8 ff.):

$\mathrm{F}(\mathrm{A})=\mathrm{W}$

Diese Formel besagt, dass die Anwendung einer bestimmten Funktion (=F) auf ein bestimmtes Argument $(=\mathrm{A})$ einen bestimmten Wert $(=\mathrm{W})$ ergibt. Wenn man ein bestimmtes Argument in einen bestimmten Zusammenhang stellt wie z.B. die Betrachtung einer Stadt unter dem Aspekt der Einwohnerzahl oder die Betrachtung eines Menschen in seiner Eigenschaft als Autor, betrachtet man das Argument in einer bestimmten Funktion. Das Ergebnis sind Werte, d.h. funktionale Einordnungen von Argumenten:

$\mathrm{F}(\mathrm{A})=\mathrm{W}$

Einwohnerzahl (Kassel) $=195.000$

Autor sein (Wolf Haas) $=$ der Autor Wolf Haas

Die Anwendung der Funktion-Argument-Wert-Formel auf die drei grammatischen Ebenen der GTA ergibt Folgendes:

Makroebene:

textgrammatische Funktion (Textsequenz) = Textglied

Mesoebene:

satzgrammatische Funktion (Satzsequenz) = Satzglied

Mikroebene:

wortgruppengrammatische Funktion (Wortgruppensequenz)

$=$ Wortgruppenglied

Eine Sequenz ist eine grammatische Form, eine Konstituente, auf der jeweiligen Ebene, der sich eine bestimmte grammatische Funktion auf derselben Ebene zuordnen lässt. Qua grammatischer Funktion erhält dann die grammatische Form ihren grammatischen Wert.

Auf Meso- und Mikroebene lässt sich mit den (relativ gut) etablierten Funktionen Subjekt, Objekt und Adverbial (Mesoebene) bzw. Kopf, Kern und Attribut (Mikroebene) arbeiten. ${ }^{2}$ Hier am Beispiel der Anwendung von zwei satzgramma-

2 Die wortgruppengrammatischen Funktionen ,Kopf‘ und ,Kern' werden in Anlehnung an Teuber (2005, S. 25 ff.) und Eisenberg (2006, S. 51 ff.) verwendet. Die Unterscheidung entspricht in etwa der zwischen lexikalischem und funktionalem Kopf in der generativen Grammatik. 
tischen Funktionen und einer wortgruppengrammatischen Funktion auf dieselbe grammatische Form, die Präpositionalgruppe auf der Startbahn:
a. Sie besteht auf der Startbahn.
b. Sie übernachteten auf der Startbahn.
c. Die Landung auf der Startbahn.

(Beispiele nach Eisenberg 2006, S. 40)

$\mathrm{F}(\mathrm{A})=\mathrm{W}$
a. Objekt (Präpositionalgruppe) = Präpositional ${ }_{\text {auf }+\mathrm{DAT}}{ }^{-o b j e k t}$
b. Adverbial (Präpositionalgruppe) = Lokaladverbial
c. Attribut (Präpositionalgruppe) = Präpositionalattribut

Die Werte sind auf der Mesoebene Satzglieder (hier: Präpositional ${ }_{\text {auf }+D A T}$-objekt, Lokaladverbial), auf der Mikroebene Wortgruppenglieder (hier: Präpositionalattribut). ${ }^{3}$

Auf der Makroebene hingegen gibt es keine etablierten (text-)grammatischen Funktionen. Deshalb mussten sie in der GTA neu eingeführt und begründet werden.

$$
\begin{aligned}
\mathrm{F}(\mathrm{A})= & \mathrm{W} \\
& + \text { Textautokod }(\text { Textsequenz })=\text { Satz } \\
& \text {-Textautokod }(\text { Textsequenz })=\text { Nichtsatz } \\
& \text { Textkohäsiv }(\text { Textsequenz })=\text { Kohäsionsglied }
\end{aligned}
$$

Die erste Makroformel besagt, dass Textsequenzen, die grammatisch autonom kodieren (= +Textautokod) den (textgrammatischen) Wert ,(grammatischer) Satz erhalten. ${ }^{4}$ Ein Satz enthält dabei ein einziges Hauptprädikat (= Prädikat eines einfachen Satzes oder Hauptsatzprädikat eines Satzgefüges) und hat qua Klammerbildungspotenzial des Hauptprädikats eine Felderstruktur. ${ }^{5}$

3 Unterschieden werden in der GTA 16, in Höllein (2019) 17 Präpositionalobjekte. Entscheidend ist dabei, dass es keinen satzgrammatischen Wert, d.h. kein Satzglied ,Präpositionalobjekt‘ gibt, weil der Begriff ,Präpositionalobjekt‘ ein abstrakterer Begriff ist als ,Akkusativobjekt‘, ,Dativobjekt' oder ,Genitivobjekt‘. ,Präpositionalobjekt‘ stellt einen Oberbegriff dar und liegt auf derselben begriffslogischen Ebene wie ,Kasusobjekt‘. Eisenbergs Analyse und die Anwendung der Formel werden in Kapitel 4 noch zu präzisieren sein. In Kapitel 6 wird gezeigt, dass bei bestehen in den letzten Jahrzehnten ein Sprachwandel $a u f{ }_{\mathrm{DAT}}>a u f{ }_{\mathrm{AKK}}$ stattgefunden hat, sodass Eisenbergs Beispiel nicht mehr dem Sprachgebrauch der jungen Generation entspricht.

4 Der Textautokod-Begriff stellt die analogische Übertragung des Konzepts der autonomen Kodierung (siehe IDS-Grammatik 1997, S. 1039 f.) auf die Makroebene dar.

5 Unter ,Satz (ohne Attribuierung) wird immer der grammatische Satz verstanden. Von diesem zu unterscheiden ist der orthografische Satz, siehe Kapitel 3. 
Dagegen stellen Nichtsätze (textgrammatische) Werte von Textsequenzen dar, die grammatisch nicht autonom kodieren. Nichtsätze können deshalb nicht autonom kodieren, weil sie keine Prädikate enthalten, die ihre Satzglied-Grundstruktur und ihre Felderstruktur festlegen und somit für eine (relative) grammatische Autonomie sorgen könnten.

Die (textgrammatische) Funktion ,Textkohäsiv“ wird auf Textsequenzen angewandt, die aus Sätzen, Nichtsätzen oder aus ganzen Textsequenzen Satz- und Nichtsatzverbindungen bzw. Textverkettungen machen. Sie können in der sogenannten Zwischenstelle zwischen Sätzen und/oder Nichtsätzen stehen (siehe Kap. 3) oder in die topologische Struktur des jeweiligen Satzes integriert sein (siehe Kap. 5). Ein Kohäsionsglied stellt also einen textgrammatischen Relator zwischen zwei Sätzen, zwischen zwei Nichtsätzen oder zwischen einem Satz und einem Nichtsatz dar.

Die drei Makrowerte (= Makroglieder/Textglieder) sollen im folgenden Kapitel kurz exemplifiziert werden.

\section{Exhaustivität auf Textebene}

Wie erwähnt, bedeutet das Streben nach phänomenbezogener deskriptiver Vollständigkeit das Streben nach Exhaustivität sowohl auf der Text- als auch auf der Satzebene. Exhaustivität auf der Textebene heißt, dass der Text vollständig in Textglieder segmentiert wird bzw. dass diese klassifiziert und zueinander in Relation gesetzt werden. Die drei Makrowerte ,Satz‘, ,Nichtsatz‘ und ,Kohäsionsglied‘, die in Kapitel 2 eingeführt wurden, sollen nun an dem folgenden (einfachen) Beispiel exemplifiziert werden: ${ }^{6}$

(2) 1. Reither sah auf die Straße,

2. er hatte das schon fast vergessen, wie gut es tun konnte, nachts neben einer Frau Auto zu fahren.

3. Und

4. von der Seite kein Wort,

5. Stille im Wagen,

6. nur das Motorgeräusch;

6 In der GTA werden folgende Markierungskonventionen verwendet, um die drei Sorten von Textgliedern optisch leicht identifizieren zu können: Bei Sätzen werden die Hauptprädikate fett markiert. Nichtsätze sind Punkt-Strich unterstrichen, Kohäsionsglieder unterstrichen. 
7. unzählige Male hatte er so die Nacht überwunden,

8. neben sich eine Schlafende,

9. irgendwie in den Sitz gekauert,

10. ein Bein bedeckt

11. und

12. eines nackt

13. und

14. auf dem nackten seine Hand.

15. $\underline{\text { Aber }}$

16. Leonie Palm war hellwach,

17. sie zündete eine Zigarette an

18. und

19. reichte sie ihm,

20. das war auch ein Wort,

21. dann rauchte sie selbst

22. und

23. öffnete etwas ihr Fenster

24. und

25. er machte seins ganz auf, weil sie schon zur Mautstelle für die Brennerautobahn kamen; (Kirchhoff Widerfahrnis, S. 55)

Wie die Nummerierung anzeigt, enthält die Textstelle insgesamt 25 Textglieder: zwölf Sätze, sechs Nichtsätze und sieben Kohäsionsglieder. ${ }^{7}$ Diese entfallen auf insgesamt zweieinhalb orthografische Sätze. ${ }^{8}$

7 Auf die Begründung und die Unterklassen kann hier nicht eingegangen werden. In der GTA gibt es zu jedem Makroglied-Typ und dessen Klassen ein eigenes Kapitel. Erwähnt werden soll an dieser Stelle lediglich, dass jede Grammatik einen wohl begründeten Prädikatsbegriff braucht. Zur Begründung des Prädikatsbegriffs der GTA gehört auch, dass das Prädikativum als Teil des Prädikats modelliert wird (siehe GTA 2017, S. 358 ff.). Wenn jemand das Prädikat ,aus dem Inventar der Satzglieder“ eliminieren will (Christ 2017, S. 170), dann müsste er auch zeigen können, wie eine exhaustive Mesoglied-Lehre, mit der man beliebige Texte grammatisch beschreiben kann, ohne ein umfangreiches Prädikatskonzept und ausschließlich unter Bezug auf die grammatische Form ,Verb` funktionieren könnte. Gerade für den schulischen Grammatikunterricht braucht man einen wert- (und nicht form-)bezogenen archimedischen Punkt für den Satz, um die Grundstruktur des Satzes verständlich machen und überhaupt den Sinn der Satzgliedanalyse vermitteln zu können.

8 Der dritte orthografische Satz, der mit Textglied Nr. 15 anfängt, endet ja mit Textglied Nr. 25 noch nicht. 
Zwar ist die Untersuchung der Relation von Textgliedern zu orthografischen Sätzen, besonders im Falle von literarischen Texten, eine lohnende Aufgabe (siehe Ágel 2015a), doch steht aus syntaktischer Sicht ein anderer Aspekt im Mittelpunkt: Würde man die Syntax auf die Satzebene reduzieren, bliebe mehr als die Hälfte des obigen Textauszugs - sieben Kohäsionsglieder und sechs Nichtsätze (vs. zwölf Sätze) - aus der syntaktischen Analyse ausgeklammert.

Was die Kohäsionsglieder anbelangt, gab es bislang keine Angebote, diesen makrosyntaktischen Wert in ein syntaktisches Modell zu integrieren, obwohl die Wörter und Ausdrücke (siehe Hennig/Buchwald-Wargenau 2010), die der Klasse zuzuordnen sind, alle bekannt sind. Dies ist insofern nicht verwunderlich, als der traditionell aszendente und an der Satzgrenze haltmachende Blick auf die Syntax Kohäsionsglieder nicht als syntaktische Werte, sondern eben nur als Wörter und Ausdrücke modellieren kann, die mehr oder weniger problematischen Wortarten oder Ausdrucksklassen angehören. Aus traditioneller Sicht wären also die - im Übrigen recht schlichten - Kohäsionsglieder des Kirchhoff-Textes nur als Wörter, als (kopulative und adversative) Konjunktoren, zu bestimmen. ${ }^{9}$ Dass dies ein elementarer theoretischer Widerspruch ist, ist leicht einzusehen: Wer auf der Makroebene Wörter als syntaktische Grundwerte akzeptiert, müsste dies auch auf der Mesoebene tun. Ein Analogon auf der Mesoebene wäre etwa, den Satz Klaus trifft Peter nicht als Subjekt-Prädikat-Akkusativobjekt, sondern als SubstantivVerb-Substantiv zu analysieren. ${ }^{10}$

Was nun die Nichtsätze anbelangt: Theoretisch bestünde höchstens die Möglichkeit, die sechs Nichtsätze als Ellipsen, als unvollständige Sätze zu analysieren, also sie allesamt auf ein Satzformat zu beziehen.

Dies dürfte einerseits syntaxtheoretisch problematisch sein, da es sich bei den sechs Nichtsätzen des Kirchhoff-Textes nicht um Koordinationsellipsen handelt. ${ }^{11}$

Andererseits wäre die Rekonstruktion von Satzformaten bei Texten, die kaum Sätze enthalten, nicht nur sprachrealitätsfern, sondern schlicht auch unmöglich. Man betrachte etwa den Anfang von Finn-Ole Heinrichs Erzählung „Soweit kein ungewohntes Wort“. Der Text beginnt mit dreizehn Nichtsätzen, erst das vierzehnte Textglied ist ein Satz:

9 Schlicht sind diese Kohäsionsglieder auch deshalb, weil sie im Gegensatz zu satzintegrierten Kohäsionsgliedern (Kap. 5) in der Zwischenstelle zwischen zwei Sätzen stehen.

10 Auf die syntaktische Notwendigkeit des Makrowerts ,Kohäsionsglied' und auf weitere Widersprüche einer aszendenten Satzsyntax kommen wir in Kapitel 4 und 5 zu sprechen.

11 Formale Syntaktiker versuchen lediglich die Koordinationsellipsen auf das Satzformat zu beziehen. Der Bezug auf das Satzformat ist zwar richtig, doch Koordinationsellipsen sind weder prosodisch noch syntaktisch elliptisch (siehe Ágel/Kehrein 2013). In der GTA werden sie als virtuelle Sätze modelliert (Textglieder Nr. 19 und 23 im Kirchhoff-Text). 
(3) 1. Sechs Schubladen insgesamt.

2. Links Stifte, Zeichenutensilien, Schere und Messer,

3. rechts Papier, Briefumschläge und Heftchen,

4. karierte, linierte und französisch linierte.

5. In der Mitte: Pfeife, Tabak, Pfeifenreiniger,

6. von allem eine beträchtliche Auswahl.

7. Darüber ein Klappfach, in dem er die Dokumente aufbewahrte,

8. Zeugnisse von Marie und mir, Geburtsurkunden, Impf- und Reisepässe, Meldebescheinigungen, den Trauschein; [...].

9. Unter der Arbeitsfläche ein Flügelklappfach,

10. gehalten von zwei kleinen Magneten,

11. geteilt von zwei Brettern in drei Kammern.

12. Oben Briefe und Postkarten,

13. darunter Steuererklärungen und Belege auf zwei Ebenen.

14. Er hat nichts mitgenommen.

(Heinrich Soweit, S. 67)

Wie ließen sich die ersten dreizehn Textglieder in einer traditionellen, aszendenten Satzsyntax beschreiben?

\section{Rekursivität, Recycling, Deszendenz}

Das Beschreibungs- und Erklärungspotenzial der Funktion-Argument-Wert-Formel erschöpft sich nicht in ,horizontalen', sich auf eine Ebene beziehenden, Anwendungen. Denn die Formel ist rekursiv: Ein erster Wert als Ergebnis der ersten Anwendung der Funktion-Argument-Wert-Formel $\left(=\mathrm{W}_{1}\right)$ kann in einem neuen Zusammenhang, d.h. bei einer zweiten Anwendung, als Argument $\left(=\mathrm{A}=\mathrm{W}_{1}\right)$ eingesetzt werden. Das Ergebnis ist ein zweiter Wert $\left(=\mathrm{W}_{2}\right)$ :

$$
\begin{aligned}
\mathrm{F}(\mathrm{A})= & \mathrm{W}_{1} \\
& \text { Autor sein (Wolf Haas })=\text { der Autor Wolf Haas } \\
\mathrm{F}(\mathrm{A}= & \left.\mathrm{W}_{1}\right)=\mathrm{W}_{2} \\
& \text { lesen (der Autor Wolf Haas })=\text { der lesende Autor Wolf Haas }
\end{aligned}
$$

Rekursivität leistet ,vertikale‘, deszendent-ebenenübergreifende, Transparenz zwischen der Erst- und der Zweitanwendung, d.h. Wiederverwendbarkeit eines primären Werts (hier: der Autor Wolf Haas) bei der Herstellung eines sekundären Werts (hier: der lesende Autor Wolf Haas). 
Überträgt man dieses rekursive Potenzial auf die drei funktionalgrammatischen Ebenen der GTA, ergibt sich daraus die Möglichkeit, syntaktische und semantische Transparenz zwischen syntaktisch analogen Strukturen auf unterschiedlichen Ebenen herzustellen, indem Werte höherer Ebenen auf niedrigeren Ebenen recycelt werden. ${ }^{12}$ Recycling bedeutet also Rekursivität zwischen funktionalgrammatischen Ebenen mit den folgenden Optionen:

1. Makroglied > Mesoglied: Makroglieder (bzw. Textsequenzen oder ganze Texte) können als Mesoglieder recycelt werden, d.h.

$$
\begin{aligned}
F(A)= & W_{1} \\
& \text { textgrammatische Funktion }(\text { Textsequen })=\text { Textglied } \\
F(A= & \left.W_{1}\right)=W_{2} \\
& \text { satzgrammatische Funktion }(\text { Textglied })=\text { Text-Satzglied }
\end{aligned}
$$

2. Makroglied $>$ Mikroglied: Makroglieder (bzw. Textsequenzen oder ganze Texte) können als Mikroglieder recycelt werden, d.h.

$$
\begin{aligned}
\mathrm{F}(\mathrm{A})= & \mathrm{W}_{1} \\
& \text { textgrammatische Funktion }(\text { Textsequenz })=\text { Textglied } \\
\mathrm{F}\left(\mathrm{A}=\mathrm{W}_{1}\right)=\mathrm{W}_{2} & \\
& \begin{aligned}
& \text { wortgruppengrammatische Funktion }(\text { Textglied })= \text { Text-Wortgruppen- } \\
& \text { glied }
\end{aligned}
\end{aligned}
$$

3. Mesoglied > Mikroglied: Mesoglieder können als Mikroglieder recycelt werden, d.h.

$$
\begin{aligned}
& \mathrm{F}(\mathrm{A})=\mathrm{W}_{1} \\
& \quad \text { satzgrammatische Funktion (Satzsequenz) = Satzglied } \\
& \mathrm{F}\left(\mathrm{A}=\mathrm{W}_{1}\right)=\mathrm{W}_{2} \\
& \quad \text { wortgruppengrammatische Funktion (Satzglied) }=\text { Satz-Wortgruppen- } \\
& \text { glied }
\end{aligned}
$$

12 Das Recycling-Konzept der GTA lehnt sich an Lucien Tesnières Translationstheorie (siehe Tesnière 1976, S. 359 ff.) an. Besonders wichtig ist dabei seine Theorie der Translation zweiten Grades („translation du second degré“) (siehe Tesnière 1976, S. 543 ff. und 1980, S. 334 ff.). Eine Andeutung, dass Recycling eine wichtige Technik in den menschlichen Sprachen ist, findet man auch bei Coseriu (1994, S. 210), der von Subordinierung spricht: „Die Subordinierung ist das Gegenteil der Superordinierung, nämlich eine allgemein gegebene Möglichkeit, die darin besteht, daß Einheiten, die konstitutionell einer bestimmten grammatischen Schicht angehören, ,im Rang zurückgestuft" werden und auf einer niedereren Ebene funktionieren“. 
Ein Beispiel für 1. ist die Wiederverwendung eines (komplexen) Satzes als Akkusativobjekt $(\mathrm{rec}=$ recycelt $):^{13}$

Ich fürchtete, (Akkusativobjekt-(Sa- wenn ich sie länger ansähe, würden kleine Tierchen hinter ihr hervorkriechen $_{\mathrm{tz} \text { ) }}^{\text {rec }}{ }_{\text {text(glied)) }}$ (Hein Freund, S. 156)

$\mathrm{F}(\mathrm{A})=\mathrm{W}_{1}$

+ Textautokod (Textsequenz) $=$ Satz

$\mathrm{F}\left(\mathrm{A}=\mathrm{W}_{1}\right)=\mathrm{W}_{2}$

Objekt (Satz) $=$ Akkusativobjekttext(glied)

Ein Beispiel für 2. ist die Wiederverwendung eines (ebenfalls komplexen) Satzes als Attribut:

Ich traute meinen Ohren nicht, was meine Gefährten da zum Besten gaben, war das Lied (Attribut-(Sa- Wem Gott will rechte Gunst erweisen, den

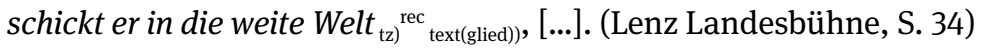

$$
\begin{aligned}
\mathrm{F}(\mathrm{A})= & \mathrm{W}_{1} \\
& + \text { Textautokod (Textsequenz })=\text { Satz } \\
\mathrm{F}(\mathrm{A}= & \left.\mathrm{W}_{1}\right)=\mathrm{W}_{2} \\
& \text { Attribut (Satz) }=\text { Attributtext(glied) }
\end{aligned}
$$

Was 3. - Mesoglied > Mikroglied - anbelangt, sollen Eisenbergs Beispiele aus Kapitel 2 - hier jedoch mit seiner Indizierung der Konstituenten - erneut aufgegriffen werden:
a. Sie [besteht $]_{\mathrm{V}}[\text { auf der Startbahn }]_{\mathrm{PrGr}}$.
b. [Sie übernachteten $]_{S}$ [auf der Startbahn $]_{\mathrm{PrGr}}$.
c. Die [Landung $]_{\mathrm{N}}[\text { auf der Startbahn }]_{\mathrm{PrG}}$.
(Eisenberg 2006, S. 40)

Eisenberg (2006, S. 41) unterscheidet zwischen dem Vor- und dem Nachbereich von syntaktischen Relationen. Angenommen wird, dass bei syntaktischen Relationen eine Konstituente $\mathrm{f}_{1}$ im Vorbereich der Relation (= eine grammatische Form) $\mathrm{zu}$ einer Konstituente $\mathrm{f}_{2}$ im Nachbereich der Relation (= zu einer anderen gramma-

13 Bei einsilbigen Termini wie ,Satz‘ umklammern rechtschreibwidrige Trennungen wie Sa-tz das fragliche Glied. 
tischen Form) in Beziehung gesetzt werde. „f $\mathrm{f}_{1}$ ist die Konstituente, um deren Funktion es geht“" (ebd.).

Der Vorbereich ist in allen drei Fällen dieselbe Konstituente, die Präpositionalgruppe (= PrGr) auf der Startbahn. Die Nachbereiche sind unterschiedlich. Eisenberg setzt die Konstituentenkategorien ,Verb` (=V), ,Satz‘ (=S) und ,Nomen“ $(=\mathrm{N})$ an. Die Präpositionalgruppe im Vorbereich hat demnach, je nachdem ob es sich um die PrGr-zu-V-, PrGr-zu-S- oder PrGr-zu-N-Relation handelt, drei verschiedene grammatische Funktionen: Präpositionalobjekt, adverbiale Bestimmung und Präpositionalattribut (siehe Eisenberg 2006, S. 40 f.).

Diese Auffassung scheint auf den ersten Blick mit der in Kapitel 2 vorgenommenen Abbildung von Eisenbergs Modell auf die Funktion-Argument-Wert-Formel im Einklang zu stehen:

$\mathrm{F}(\mathrm{A})=\mathrm{W}$
a. Objekt (Präpositionalgruppe) = Präpositional ${ }_{\text {auf }+ \text { DAT }}{ }^{-o b j e k t}$
b. Adverbial (Präpositionalgruppe) = Lokaladverbial
c. Attribut (Präpositionalgruppe) = Präpositionalattribut

Doch die Ersatzprobe bestätigt diese vorläufige Rekonstruktion nicht:
a. Sie besteht darauf.
b. Sie übernachteten hier/da/dort.
c. Die Landung hier/da/dort.

Im Allgemeinen fällt auf, dass nicht die Präpositionalgruppen als grammatische Formen, sondern deren grammatische Werte anadeiktisiert werden müssen, was unabhängig von Eisenberg oder der GTA ein sehr starkes Argument für funktionale Grammatiken ist.

Im Besonderen fällt auf, dass Eisenbergs Form-zu-Form-Modell - und somit auch meine vorläufige Rekonstruktion - zwar die unterschiedlichen Werte ,Prä-

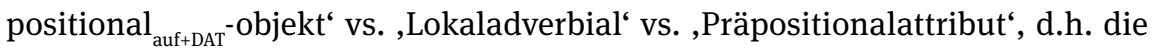
,horizontalen`Differenzen zwischen a, b und c erklärt: identische Formen mit unterschiedlichem Werten. Sie erklärt aber nicht, woher die ,vertikalen' Gemeinsamkeiten zwischen b und c, d.h. die, vertikalen' Differenzen zwischen a (darauf) auf der einen Seite und b und c (hier/da/dort) auf der anderen, kommen. Denn, wie die Ersatzprobe zeigt, der Adverbialwert auf Mesoebene und der Attributwert auf Mikroebene werden identisch anadeiktisiert. Dies muss ein syntaktisches Modell auch abbilden können.

Um solche ,vertikalen' Analogien in den Griff zu bekommen, muss die rekursive Anwendbarkeit der Funktion-Argument-Wert-Formel genutzt werden: 
$\mathrm{F}(\mathrm{A})=\mathrm{W}_{1}$

b. Adverbial (Präpositionalgruppe) = Lokaladverbial

$\mathrm{F}\left(\mathrm{A}=\mathrm{W}_{1}\right)=\mathrm{W}_{2}$

c. Attribut (Lokaladverbial) $=$ Lokal(adverbial)attribut

Die ,vertikalen' Gemeinsamkeiten zwischen b und c sind also nicht zufällig, ausschließlich formbezogen sind sie jedoch nicht zu erfassen. Dasselbe gilt für das Präpositional $_{\text {auf }+ \text { DAT }}$-objekt, das sich als Präpositional ${ }_{\text {auf }+D A T}-$ objektattribut recyceln lässt:

(6”) a. Sie besteht auf der Startbahn/darauf.

d. Ihr Bestehen auf der Startbahn/darauf.

$\mathrm{F}(\mathrm{A})=\mathrm{W}_{1}$

a. Objekt (Präpositionalgruppe) $=$ Präpositional $_{\text {auf }+\mathrm{DAT}}$-objekt $\mathrm{F}\left(\mathrm{A}=\mathrm{W}_{1}\right)=\mathrm{W}_{2}$

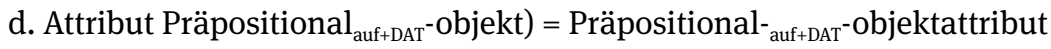

Daraus folgt, dass auch der traditionelle formale Begriff des Präpositionalattributs inadäquat ist: Bei recycelten Adverbialen stellt eine bestimmte Präpositionalgruppe nur eine von vielen möglichen Realisierungen des adverbialen Werts dar: die Landung darauf/auf/neben/vor der Startbahn/hier/da/dort. Bei recycelten Präpositionalobjekten hingegen ist sie die einzig mögliche Realisierung des Präpositionalobjektwerts: das Bestehen darauf/auf $/{ }^{*}$ neben $/{ }^{*}$ vor der Startbahn $/{ }^{*}$ hier $/{ }^{*}$ da $/{ }^{*}$ dort.

Im Lichte des Recycling-Konzepts sind aber nicht nur die traditionellen Attributwerte zu korrigieren, sondern auch die Wortart ,Präpositionaladverb‘. Es stellt sich nämlich die Frage, warum anadeiktisches darauf nur dann mit einem anderen anadeiktischen Adverb kommutiert, wenn beide einen - genuinen oder recycelten - Adverbialwert haben.

(6"') a-d. Sie besteht/Ihr Bestehen darauf/*dort.

b-c. Sie übernachteten/Die Landung darauf/dort.

Hier führt die aszendente, ,von unten nach oben'-Perspektive theoretisch in eine Sackgasse: Man ordnet vor der Satzgliedbestimmung jedem Wort eine Wortart zu, die dann der Satzgliedbestimmung zugrunde liegt. Doch lassen sich Objekte im Allgemeinen und Präpositionalobjekte im Besonderen durch keine Adverbien realisieren. Dagegen kann ein Adverbial sowohl durch ein Adverb als auch durch (diverse) Präpositionalgruppen realisiert werden: 
$\mathrm{F}(\mathrm{A})=\mathrm{W}$

a. Objekt (Präpositionalgruppe/ ${ }^{\star}$ Adverb) = Präpositional- ${ }_{\text {auf }+ \text { DAT }}{ }$ objekt

b. Adverbial (Präpositionalgruppe/Adverb) = Lokaladverbial

Die nominale Realisierungsform eines Präpositionalobjekts ist die Präpositionalgruppe. Grafische Wörter wie darauf, dagegen, danach usw. sind demnach syntaktisch keine Wörter, sondern Pro-Präpositionalgruppen, d.h. deiktische Minimalformen von Präpositionalgruppen. Auch in den Fällen, in denen die ProPräpositionalgruppe adverbialen Satzgliedwert hat, d.h. Adverbialbestimmung ist, bleibt sie ja eine Präpositionalgruppe und mutiert nicht zu einem Adverb.

Die sogenannten Präpositionaladverbien stellen also syntaktisch keine Wortart, keine Adverbsubklasse dar, sondern sie gehören der Wortgruppenart ,Präpositionalgruppe‘ an: Sie stellen die deiktische Realisierungsform von Präpositionalgruppen dar.

Die Erörterungen in diesem Kapitel sollten exemplifizieren, was unter einer deszendenten Syntax zu verstehen ist:

1. Theoretisch bedeutet Deszendenz Recycling. In einer deszendenten Syntax werden grammatische Werte nicht nur horizontal, auf derselben Ebene, modelliert, sondern sie werden auch vertikal von oben nach unten ,gereicht". Durch grammatisches Recycling, das sprachenübergreifend einen Ökonomiefaktor darstellen dürfte, werden syntaktisch-semantische Zusammenhänge zwischen den drei funktionalgrammatischen Ebenen sichtbar gemacht.

2. Methodisch bedeutet Deszendenz die Vorgehensweise bei jeder Textanalyse: von der Makro- über die Meso- bis hin zur Mikroebene. Dieses Vorgehen hat theoretische Konsequenzen, weil Beschreibungs- und Erklärungsoptionen ins Blickfeld rücken, die aszendent wohl nicht erschließbar gewesen wären. Hierzu gehören die Wörter ohne Wortart, d.h. die Präpositionaladverbien genannten Pro-Präpositionalgruppen, aber auch die satzinternen Kohäsionsglieder, die im folgenden Kapitel behandelt werden sollen.

\section{Restlosigkeit der Satzanalyse}

Das Streben nach phänomenbezogener deskriptiver Vollständigkeit (Kap. 1) bedeutet nicht nur Exhaustivität auf Textebene (Kap. 3), sondern auch Exhaustivität auf Satzebene: In einer funktionalen Satzanalyse müssen allen grammatischen Formen in einem Satz Werte zugewiesen werden können; die Analyse muss also dem Kriterium der Restlosigkeit der Satzanalyse genügen. 
Genau das ist jedoch in einer ,Von unten nach oben'-Satzanalyse, die mit grammatischen Verlusten einhergeht, nicht der Fall: In Sätzen lassen sich die Wörter in der Regel zwar Wortarten zuordnen, aber zahlreiche Wörter - und auch Ausdrücke - gehen auf dem Wege von den Wortarten zu den Satzgliedern (oder Gliedteilen) ,verloren': Abtönungspartikeln, Fokuspartikeln, die Negationspartikel, sogenannte Textadverbien und satzintegrierte Junktoren. In Grammatiken sucht man vergeblich nach einer Antwort etwa auf die Frage, wie Abtönungspartikeln auf der Satzebene einzuordnen sind. Somit ergibt sich auch hier ein vergleichbarer theoretischer Widerspruch wie bei Kohäsionsgliedern in der Zwischenstelle (Kap. 3): Aszendent könnte man auf der Mesoebene einen Satz wie Klaus trifft ja Peter nur als Subjekt-Prädikat-Abtönungspartikel-Akkusativobjekt beschreiben.

In der GTA werden Abtönungspartikeln, Fokuspartikeln, satzintegrierte Junktoren und Textadverbien als Kohäsionsglieder, d.h. als Textgliederim Satz, modelliert: ${ }^{14}$

Warum dieser Schreck, den er noch in allen Gliedern spürte? Es ist (Abtönungs- $\underline{\text { doch }}_{\text {partikel) }}$ nichts als eine alte dumme Geschichte [...]. (Werfel Blassblau, S. 18)

Sie [= die Minister, VÁ] besaßen keinen rechten Einblick in die Laby-

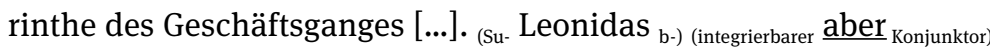
(je- und seinesgleichen ${ }_{\mathrm{kt} \text { ) }}$ hatten das Regieren gelernt wie Musiker den Kontrapunkt lernen in jahrelang unablässiger Übung. Sie besaßen ein nervöses Fingerspitzengefühl für die tausend Nuancen des Verwaltens und Entscheidens. Die Minister spielten (in ihren Augen) (Fokus- $\underline{\text { nur }}_{\text {partikel) }}$ die Rolle politischer Hampelmänner [...]. (Werfel Blassblau, S. 67)

Die Abtönungspartikel doch in (7) verbindet den Warum-Nichtsatz mit dem darauf folgenden Satz. Ihre kohäsive Leistung lässt sich mit der eines konzessiven Junktors vergleichen.

In dem Beleg (8) gibt es gleich zwei Kohäsionsglieder: Der integrierte (adversative) Konjunktor aber verbindet den ersten mit dem zweiten Satz, die Fokuspartikel nur, die ebenfalls adversativ funktioniert, den dritten mit dem vierten.

Besonders aufschlussreich ist die Stellung des Konjunktors aber. Er ist in das Subjekt des zweiten Satzes (Leonidas und seinesgleichen) integriert, gehört aber weder zum Subjekt, das hier als ein „Koordinatepaar“ (HdK 2003, S. 268) reali-

14 Die Textadverbien, auf die hier nicht eingegangen wird, heißen Ordnungsglieder und gehören der Subklasse der Konnektoren an. Die Negationspartikel wird als Kommentarglied, ein Satzgliedtyp auf Mesoebene, rekonstruiert. 
siert wird, noch zu einem anderen Mesoglied. Für diese Stelle nach dem ersten „Koordinat“ (ebd.), d.h. nach Leonidas, ist selbst in dem differenzierten Felderstrukturmodell des HdK 2003 keine Stelle vorgesehen. Vielmehr stellt der Belegtyp das Konzept der Nacherstposition (siehe ebd., S. $71 \mathrm{f}$.) infrage. ${ }^{15}$ Man vergleiche den Originalbeleg mit den folgenden Varianten mit und ohne Koordinatepaar:

Sie besaßen keinen rechten Einblick in die Labyrinthe des Geschäfts-

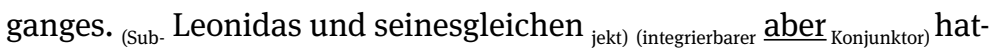
ten das Regieren gelernt wie Musiker den Kontrapunkt lernen in jahrelang unablässiger Übung.

Sie besaßen keinen rechten Einblick in die Labyrinthe des Geschäfts-

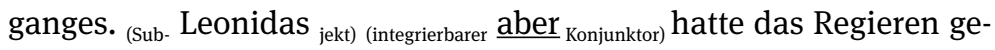
lernt wie Musiker den Kontrapunkt lernen in jahrelang unablässiger Übung.

Das integrierte Kohäsionsglied aber kann seine kohäsive Leistung erbringen, indem es nach einer potenziell mesogliedfähigen Textsequenz platziert wird. Diese kann ein einfaches Satzglied, das erste Koordinat eines Koordinatepaars oder ein Koordinatepaar sein.

Beleg (8) exemplifiziert übrigens einen Typ, der in der recht umfangreichen Literatur zur sogenannten mehrfachen Vorfeldbesetzung nicht vorzukommen scheint. ${ }^{16}$ Genauso wenig wie der folgende Typus:

Von seiner »sozialen Entbindung« überschätzt der junge bürgerliche Mensch die Schwierigkeit des Sprunges in die Welt. (Sub- Ich jekt) (Fokuspersönlich partikel) (Nacherst. zum Beispiel $_{\text {Adverbjunktor) }}$ verdankte meine erstaunliche Karriere durchaus keinen überragenden Eigenschaften, sondern drei musikalischen Talenten [...]. (Werfel Blassblau, S. 52)

Hier verbinden gleich zwei Kohäsionsglieder den ersten mit dem zweiten Satz: die Fokuspartikel persönlich, deren kohäsive Leistung sich als Individuierung der junge bürgerliche Mensch > ich - beschreiben lässt, und der Nacherst-Adverbjunktor zum Beispiel, der exemplifizierend funktioniert. ${ }^{17}$

15 Die Kritik betrifft auch die GTA, in der mit dem Konzept ebenfalls gearbeitet wurde.

16 Einen materialreichen Überblick bieten Bassola/Schwinn (2016).

17 In dem Korpus von Bassola/Schwinn (2016, S. 251) kommen Satzglieder im Vorfeld entweder mit einer „Rangierpartikel“ wie beispielsweise oder mit einer Fokuspartikel vor. 
Kurzes Fazit: In Sätzen kommen nicht nur Satzglieder, sondern auch Textglieder vor. Eine funktionale Satzanalyse mit dem Anspruch, alle grammatischen Werte, die in einem Satz vorkommen, zu identifizieren, bedeutet also, dass auch die satzintegrierten Kohäsionsglieder identifiziert werden müssen.

\section{Grammatische Grundstruktur als semantischer Differenzwert}

Einzelsprachliche Grammatik und einzelsprachliche Semantik sind keine autonomen Größen (siehe Coseriu 1987). Die Wahl einer bestimmten grammatischen Struktur in einer Sprache - genauer: Vollvarietät - impliziert immer auch die Wahl einer bestimmten semantischen Struktur (und die Abwahl alternativer Strukturen). Grammatische Strukturen sind also Zeichen mit einer Form- und einer Inhaltsseite. Entsprechend sind auch Satzbaupläne, die die grammatisch-semantischen Grundstrukturen von Sätzen einer Einzelsprache repräsentieren, Zeichen.

Die grammatische Grundstruktur eines Satzes besteht aus dem Hauptprädikat und dessen Komplementen. Semantisch stellt ein Satz einen qua Hauptprädikat entworfenen und qua Hauptprädikat und Komplementen realisierten einzelsprachlichen Sachverhalt, ein Szenario (siehe Fischer 2003, S. 28 ff.), dar. Dabei ist unter ,Semantik‘ strikt ,signifikative Semantik‘ zu verstehen. Signifikativ-semantisch werden - im Gegensatz zur herkömmlichen denotativen Semantik - keine außer(einzel)sprachlichen Situationen, sondern eben Szenarios, einzelsprachlich perspektivierte Sachverhalte beschrieben (Welke 2005, S. 95 ff.). ${ }^{18}$

Doch in welchem Sinne genau sind grammatische Grundstrukturen semantisch?

Sie sind nicht in dem Sinne semantisch, dass Satzglieder oder gar Kasus eigene Bedeutungen hätten, sondern in dem Sinne, dass sie einen semantischen Differenzwert (valeur) haben. Dies ist das Thema des vorliegenden Kapitels und soll unter drei Aspekten kurz beleuchtet werden:

1. Das System grammatischer Grundstrukturen hat semantischen Differenzwert.

2. Jede einzelne grammatische Grundstruktur hat semantischen Differenzwert.

18 Signifikativ-semantische Grundstrukturen - inkl. der semantischen Subjektrollen HANDLUNGSTRÄGER, VORGANGSTRÄGER und ZUSTANDSTRÄGER - wurden bereits in Fleischmann (1985) entwickelt und angewandt, ohne dass dies in der Linguistik rezipiert worden wäre. Für den Hinweis danke ich Dagobert Höllein. 
3. Der Wechsel der grammatischen Grundstruktur indiziert den Wechsel der semantischen Grundstruktur (Neuerung oder Wandel).

\section{ad 1.:}

Betrachten wir einen Satz und eine mögliche alternative Formulierung mit der Beschreibung ihrer Grundstrukturen: ${ }^{19}$

Ich schicke voraus, daß ich kein Kunstkenner bin, sondern Laie. Ich habe oft bemerkt, daß mich der Inhalt eines Kunstwerkes stärker anzieht als dessen formale und technische Eigenschaften, auf welche doch der Künstler in erster Linie Wert legt. Für viele Mittel und manche Wirkungen der Kunst fehlt mir eigentlich das richtige Verständnis. Ich muß dies sagen, um mir eine nachsichtige Beurteilung meines Versuches zu sichern. (Freud Moses, S. 172)

$\begin{array}{lll}\text { Dativobjekt } & \text { - Prädikat }- & \text { Subjekt } \\ \text { ZUSTANDSBETROFFENER } & \text { - ZUSTAND }- & \text { ZUSTANDSTRÄGER } \\ \text { mir } & \text { fehlt } & \text { das richtige Verständnis für... }\end{array}$

(10') Für viele Mittel und manche Wirkungen der Kunst bringe ich eigentlich kein richtiges Verständnis auf.

$\begin{array}{lll}\text { Subjekt } & \text { - Prädikat }- & \text { nominales Akkusativobjekt } \\ \text { HANDLUNGSTRÄGER } & \text { - HANDLUNG }- & \text { HANDLUNGSGEGENSTAND } \\ \text { ich } & \text { bringe ... auf } & \text { kein richtiges Verständnis für ... }\end{array}$

Im Originalbeleg nimmt Freud keine Handlungsperspektive ein. Mithilfe des FEHLEN-Szenarios und der grammatischen Grundstruktur ,Dativobjekt - Prädikat - Subjekt‘ positioniert er sich als ZuSTANDSBETROFFENER, als jemand, der sich selbst beobachtend feststellen muss, dass er über eine bestimmte innere Disposition nicht verfügt, dass er eben kein ZUSTANDSTRÄGER ist.

Diese Szenierung (= einzelsprachliche Sachverhaltsperspektivierung) passt gut zur Captatio Benevolentiae, die hier von Freud angestrebt wird. Die alternative Handlungsperspektivierung mithilfe des AUFBRINGEN-Szenarios hätte weniger gut gepasst.

Szenierungsalternativen lassen sich nicht nur lexikalisch - durch Hauptprädikatswechsel -, sondern auch grammatisch herstellen:

19 Die semantische Grundstruktur (= semantisches Prädikat + signifikativ-semantische Rollen) wird durch Kapitälchen gekennzeichnet. 
(11)

Jetzt hat sich die Witwe sofort wieder beruhigt.

(Haas Silentium, S. 80)

$\begin{array}{ll}\text { Subjekt } & \text { - Prädikat - } \\ \text { ENDO-VORGANGSTRÄGER } & \text { - ENDO-VORGANG - } \\ \text { Die Witwe } & \text { beruhigt sich }\end{array}$

(11') Der Brenner beruhigt die Witwe.

$\begin{array}{lll}\text { Subjekt } & \text { - Prädikat }- & \text { Akkusativobjekt } \\ \text { HANDLUNGSTRÄGER } & \text { - HANDLUNG }- & \text { HANDLUNGSGEGENSTAND } \\ \text { Der Brenner } & \text { beruhigt } & \text { die Witwe }\end{array}$

(11”) Die Witwe wird (vom Brenner) beruhigt.

$\begin{array}{lll}\text { Subjekt } & & \text { - Prädikat }- \\ \text { Exo-Vorgangsträger } & \text { - Exo-Vorgang }- & \text { (Exo-Vorgangsauslöser) } \\ \text { Die Witwe } & \text { wird beruhigt } & \text { (vom Brenner) }\end{array}$

Die drei Szenarios - das Sich-Beruhigen-, das Beruhigen- und das BerUhigtWERDEN-Szenario - stellen drei verschiedene Perspektivierungen derselben Situation dar. Je nachdem welche semantische Rolle das Subjekt indiziert, wird die Situation aus der Perspektive des HANDLUNGSTRÄGERS oder aus der von zwei verschiedenen VORGANGSTRÄGERN szeniert. ${ }^{20}$

Wie die Beispiele andeuten, lässt sich signifikativ-semantisch das Potenzial grammatischer Grundstrukturen quasi kontrastiv beschreiben. Es geht nicht, wie bei der denotativen Semantik, um die grammatische Umordnung derselben außer(einzel)sprachlichen semantischen Rollen, mit denen die Autonomie der Semantik geschickt vorgetäuscht wird, sondern um das System einzelsprachlich realisierbarer Satzbauplanzeichen: Die semantische Leistung jedes einzelnen Satzbauplanzeichens einer Einzelsprache unterscheidet sich von der jedes anderen.

20 Um die Unterschiede zwischen passivischer und medialer Realisierung zu erfassen, wurden in der GTA in Anlehnung an Haspelmath (1993, S. 108) zwei Typen von sprachlichen Perspektivierungen von Zustandsveränderungen eingeführt: exo- und endoaktiv: In ,exoaktiver Perspektive (kurz: EXO) wird eine Zustandsveränderung als äußere Einwirkung, als die Einwirkung einer externen Kraft (eines ,Auslösers`) dargestellt. Dies ist per definitionem der Fall in Handlungssätzen (mit Agenssubjekt), aber auch in passivischen Vorgangssätzen. Umgekehrt heißt ,endoaktiv` (kurz: ENDO), dass eine Zustandsveränderung einzelsprachlich als ein spontanes Geschehen, als ein Geschehen ohne äußere Einwirkung, wiedergegeben wird. Bei ENDO-Vorgängen kann es also keinen VorgangSAUSLÖSER geben. 


\section{ad 2.:}

Dass jede einzelne grammatische Grundstruktur semantischen Differenzwert hat, bedeutet, dass die semantische Grundstruktur desselben Satzbauplanzeichens auch dann erhalten bleibt, wenn die lexikalischen Besetzungen der Komplemente inkongruent sind. Z.B. wenn man die Besetzungen der Dativobjekt- und der Subjektstelle des Freud-Satzes vertauscht:

(10’) Dem richtigen Verständnis für viele Mittel und manche Wirkungen der Kunst fehle ich.

$\begin{array}{lll}\text { Dativobjekt } & - \text { Prädikat }- & \text { Subjekt } \\ \text { ZUSTANDSBETROFFENER } & - \text { ZUSTAND }- & \text { ZUSTANDSTRÄGER } \\ \text { dem richtigen } & \text { fehle } & \text { ich } \\ \text { Verständnis für ... } & & \end{array}$

Die grammatische Grundstruktur macht trotz Inkongruenz eindeutig, wer (/was) ZUSTANDSBETROFFENER und was (/wer) ZUSTANDSTRÄGER ist (siehe Knobloch 1990, S. 186). Der Inhalt des Originalsatzes hat sich zwar radikal geändert, aber die semantische Differenz zwischen ZuSTANDSBETROFFENEM und ZuSTANDSTRÄGER ist erhalten geblieben. Daraus lässt sich das Prinzip des Einmaleins der Satzgliedlehre (siehe GTA 2017, S. 493 ff.), der Grundsatz, dass dasselbe Satzglied in demselben Satz nur einmal vorkommen darf, ableiten. Schließlich setzt erfolgreiche Verständigung voraus, dass Szenariobeteiligte grammatisch einmalig kodiert und somit semantisch unterscheidbar sind.

\section{ad 3.:}

Dass der Wechsel der grammatischen Grundstruktur den Wechsel der semantischen Grundstruktur indiziert, manifestiert sich valenzdynamisch einerseits in (in den Texten permanent stattfindenden) Neuerungen, andererseits im Sprachwandel (siehe Coseriu 1974, S. 67 ff.; Ágel 2000, S. 268 ff.).

Neuerung bedeutet, dass sich ein Prädikat, das konventionell dem Satzbauplan A zuzuordnen ist, okkasionell mit einem Satzbauplan B verbindet:

Im Gegensatz zum Medellin-Kartell war das Cali-Kartell vertikal organisiert und definierte sich auf gemeinsame Kooperationen. (9.12.2014 http://de.wikipedia.org/wiki/Cali-Kartell)

Das Hauptprädikat sich definieren bedient sich hier des Satzbauplans, dem konventionell Verben wie hoffen oder sich freuen $a u f_{+\mathrm{AKK}}$ angehören. Entsprechend übernimmt das Präpositional ${ }_{\text {auf }+\mathrm{AKK}}$-objekt, das ein „zukünftiges Ereignis“ kodiert, die semantische Rolle Prospectum (siehe Höllein 2019, S. 179-181). 
Scheinbar derselbe Fall liegt im folgenden Beleg vor:

Er [= Krüger, VÁ] fuhr zu den Ziegelwerkern hinaus. »Ihr macht hier viel, aber schlechte Ziegel, meine Freunde.« »Hoho, was für Weisheiten!« die Ziegler erboten sich, gute, dafür aber weniger Ziegel zu machen. »Alle Tage, Sportsfreund!« Krüger bestand auf viel Ziegel und gute Ziegel, aber in dieses Paradies schien kein Weg zu führen. (Strittmatter Bienkopp, S. 264)

Doch hier geht es nicht um eine okkasionelle Innovation, sondern um den Sprachwandel bestehen $n_{a u f+\mathrm{DAT}}>a u f_{+\mathrm{AKK}}$. Obwohl in den Referenzwerken (fast) nur bestehen $_{\text {auf }+\mathrm{DAT}}$ kodiert ist, zeigen Tests mit jungen ProbandInnen, dass bestehen $n_{a u f+\mathrm{DAT}}$ nicht nur veraltet ist, sondern von der jungen Generation mehrheitlich sogar abgelehnt wird. Die folgende Tabelle fasst das Ergebnis eines Kasseler Tests zusammen (+ = „gut“, m = „möglich“, - = „,schlecht“): ${ }^{21}$

Tab. 2: Testergebnisse zu bestehen auf $f_{+\mathrm{AKK}} /$ auf $f_{+\mathrm{DAT}}$

\begin{tabular}{lll}
\hline URTEILE & AKKUSATIV & DATIV \\
\hline$\sum$ (absolut): & $+(233) ; \mathrm{m}(19) ;-(20)$ & $+(36) ; \mathrm{m}(49) ;-(187)$ \\
$\sum(\%):$ & $+(85,7 \%) ; \mathrm{m}(7 \%) ;-(7,3 \%)$ & $+(13,2 \%) ; \mathrm{m}(18 \%) ;-(68,8 \%)$ \\
\hline
\end{tabular}

Das Präpositional ${ }_{\text {auf }+D A T}$-objekt der älteren Generation trägt nach Höllein (2019, S. 190 f.) die semantische Rolle BAsIs, deren Bedeutung er als ,Grundlage des Szenarios‘ paraphrasiert. Der Wandel der grammatischen Grundstruktur mit Präpositional $_{\text {uuf }+\mathrm{DAT}}$-objekt $>$ Präpositional ${ }_{\text {auf }+A K K}$-objekt indiziert also den semantischen Wandel BASIS > PROSPECTUM.

21 Zu Details des 2012 in Kassel mit 23 muttersprachlichen Studierenden durchgeführten Tests, bei dem auch Eisenbergs Beispiel Die Regierung besteht auf der neuen Startbahn (Eisenberg 2006, S. 39) getestet wurde, siehe GTA 2017, S. 537 f. Der Kasseler Test wurde 2018 in Leipzig mit 18 muttersprachlichen Studierenden wiederholt. Die Ergebnisse waren im Wesentlichen identisch. Ich danke Mathilde Hennig (Gießen), die die Testwiederholung initiiert, Sandra Döring (Leipzig), die den Test durchgeführt, und Vanessa Langsdorf (Gießen), die ihn ausgewertet hat. Aufschlussreich ist der Umstand, dass eine Recherche von Volker Emmrich (Gießen) im Dudenkorpus im Rahmen der Vorbereitung der 8. Auflage von Duden 9 zum diametral entgegengesetzten Ergebnis gekommen ist: $a u f_{+\mathrm{DAT}}-$ Annotation $=$ ca. $83 \%$, auf $f_{+\mathrm{AKK}}-$ Annotation $=$ ca. $17 \%$. Es fragt sich also, inwiefern große Korpora in der Lage sind, rezenten Sprachwandel abzubilden. 


\section{Statik und Dynamik}

Der letzte Theoriebaustein, auf den hier kurz eingegangen werden soll, ist die Unterscheidung zwischen statischen und dynamischen Sätzen.

Die Grundstruktur eines Satzes basiert auf der konventionalisierten, im Lexikoneintrag des prädikatsstiftenden Verbs, Adjektivs oder Substantivs festgehaltenen Grundvalenz (z.B. Welke 2011, S. 167 ff.; 2015).

Wenn die Grundvalenz 1:1 realisiert wird, wenn also das in der Grundvalenz angelegte Szenario ohne Abwandlung, ohne Umszenierung, aktualisiert wird, entstehen statische Sätze mit statischer Valenzrealisierung (Hauptprädikate fett markiert): ${ }^{22}$

(14a) Er hat »mutmaßlich« etwas vergessen.

(15a) Er hat geschrien.

(16a) Erst vom Major aufwärts stinkt man nicht.

In den Originalbelegen wurden jedoch die Grundvalenzen der Verben vergessen, schreien und stinken nicht 1:1 realisiert, die jeweiligen statischen Szenarios wurden umszeniert:

(14b) Er hat »mutmaßlich« ein bißchen Geld an der Steuer vorbeivergessen. (Hildebrandt achtzig, S. 42)

(15b) Er hat seine eigenen Zweifel niedergeschrien. (Timm Sommer, S. 207)

(16b) Erst vom Major aufwärts wird nicht gestunken. (Lenz Überläufer, S. 203)

Die Umszenierung erfolgte in (14b) und (15b) durch Valenzträgererweiterung (vergessen > vorbeivergessen, schreien > niederschreien), in (16b) durch Passivierung (stinken $>$ wird gestunken). ${ }^{23}$

Solche Sätze, die konventionell oder kreativ sein können (Ágel 2015b), stellen dynamische Sätze mit dynamischer Valenzträger- und partiell dynamischer Valenzrealisierung dar. Dynamisch sind in allen drei Sätzen die Prädikate, darüber hinaus das Präpositional ${ }_{\mathrm{an}+\mathrm{DAT}}$-objekt an der Steuer und das Akkusativobjekt seine eigenen Zweifel. ${ }^{24}$

22 Dasselbe Verb kann mehrere Grundvalenzen haben, d.h., es gibt keine 1:1-Relation zwischen Verb und Grundvalenzträger (siehe Ágel 2000, S. 113 ff.; Welke 2015). Das Kriterium für einen Grundvalenzträger ist, dass er die Grundlage für Umszenierungen, beispielsweise für Valenzreduktionen und -erhöhungen, bilden können muss.

23 Zu den zahlreichen Formen der Prädikatsdynamik siehe Ágel (2015b) und GTA (2017, S. 399 ff.). 24 In der GTA sind dynamische Prädikate und Satzglieder türkis markiert. 
Valenz(träger)dynamik lässt sich mithilfe der Funktion-Argument-Wert-Formel abbilden. Die allgemeine Formel lautet: ${ }^{25}$

$$
\begin{aligned}
\mathrm{F}(\mathrm{A})= & \mathrm{W} \\
& \mathrm{F}\left(\mathrm{Satz}_{\text {stat }}\right)=\mathrm{Satz}_{\text {dyn }}
\end{aligned}
$$

Je nachdem ob die Funktion, die auf den statischen Satz angewandt wird, eine Konstruktion oder eine Kategorie ist, lassen sich konstruktionell und kategorial dynamische Sätze als zwei grundlegende Typen von Satzwerten unterscheiden:

$\{$ Konstruktion $\}\left(\mathrm{V}(\mathrm{TR})_{\text {stat }}\right)=$ konstruktionell $\mathrm{V}(\mathrm{TR})_{\text {dyn }}$

\{vorbei-Konstruktion $\}\left(\mathrm{V}(\mathrm{TR})_{\text {stat }}\right.$ vergessen $)=\mathrm{V}(\mathrm{TR})_{\text {dyn }}$ vorbeivergessen

$\{$ nieder-Konstruktion $\}\left(\mathrm{V}(\mathrm{TR})_{\text {stat }}\right.$ schreien $)=\mathrm{V}(\mathrm{TR})_{\mathrm{dyn}}$ niederschreien

Kategorie $\left(\mathrm{V}(\mathrm{TR})_{\text {stat }}\right)=$ kategorial $\mathrm{V}(\mathrm{TR})_{\text {dyn }}$

subjektloses Passiv $\left(\mathrm{V}(\mathrm{TR})_{\text {stat }}\right.$ stinken $)=\mathrm{V}(\mathrm{TR})_{\mathrm{dyn}}$ gestunken werden

Die Unterscheidung von statischen und dynamischen Sätzen hat weitreichende Folgen für die Satzgliedlehre, die sich gewissermaßen verdoppelt: Unterschieden und ausführlich beschrieben werden in der GTA statische und dynamische Prädikate und statische und dynamische Satzglieder.

Durch diese Unterscheidung ist es u.a. möglich, dem Prinzip des Einmaleins der Satzgliedlehre auch in den Fällen zu genügen, wo es scheinbar verletzt wird:

$$
\begin{aligned}
& \text { [...] und [er] schob } \text { (statisches }_{\text {das Fahrrad }} \text { Akkusativobjekt) }_{\text {schräg (dynamisches }} \text { den }
\end{aligned}
$$

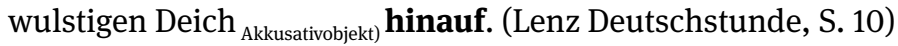

$$
\begin{aligned}
& \text { Ich sehe }_{\text {(dynamisches }} \text { ihn }_{\text {Akkusativobjekt) (statisches }} \text { die Einfahrt }{ }_{\text {Akkusativobjekt) }} \text { ausbessern. } \\
& \text { Der Inspektor lässt } \text { (dynamisches }_{\text {die Polizisten }} \text { Akkusativobjekt) (statisches } \text { die Verbre- } \\
& \text { cher }_{\text {Akkusativobjekt) }} \text { entwaffnen. }
\end{aligned}
$$

Hier liegen konstruktionell dynamische Prädikate mit zwei Akkusativobjekten einem statischen und einem dynamischen - vor. In der Grundvalenz der den Konstruktionen zugrunde liegenden statischen Valenzträger (schieben, ausbessern, entwaffnen) ist jeweils nur ein (statisches) Akkusativobjekt verankert. Es ist erst die Anwendung der jeweiligen Konstruktion auf die statischen Valenzträger, die die dynamischen Akkusativobjektwerte des Komplexverbs (hinaufschieben), des AcI- und des lassen-Prädikats erzeugt.

$25 \mathrm{Satz}_{\mathrm{stat}}=$ statischer Satz, Satz $\mathrm{dyn}=$ dynamischer Satz, V(TR) = Valenz(träger). 


\title{
8 Schluss
}

Für ein echtes Fazit ist kein Raum mehr geblieben. Es kann aber festgestellt werden, dass die IDS-Grammatik (1997, S. 4) Recht hat, wenn sie darauf hinweist, dass das Postulat (b) - die theoretische Fundierung - ,prinzipiell nicht voll eingelöst werden (kann). Vielmehr gilt das Hase-Igel-Paradox:“

\begin{abstract}
Wie gut theoretisch fundiert auch immer die Systematisierung sprachlicher Phänomene in einer Grammatik sein mag - sie fällt notwendig hinter den sich entwickelnden Forschungsstand zurück, weil die konzeptionellen Grundentscheidungen in der konkreten Ausarbeitung nicht mehr revidierbar sind und damit auch die Zugriffsweise auf Einzelphänomene prinzipiell präjudiziert ist. (IDS-Grammatik 1997, S. 5)
\end{abstract}

In der Tat musste ich in den langen Jahren der Fertigstellung des recht umfangreichen Buches feststellen, dass das theoretische Korsett, das hier kurz vorgestellt wurde, bei manch einer Beschreibung oder Erklärung eng geworden ist. Trotzdem bleibt es zu hoffen, dass die Beschreibungs- und Erklärungsansätze der GTA ausbaufähig sind, sodass die Arbeiten, die Theoriebausteine der GTA verwenden, das theoretische Korsett lockern können.

\section{Literatur}

\section{Textquellen}

Freud Moses = Freud, Sigmund (1981): Der Moses des Michelangelo. In: Freud, Sigmund:

Gesammelte Werke. Bd. 10. Werke aus den Jahren 1913-1917. Frankfurt a.M., S. 172-201.

Haas Silentium = Haas, Wolf (1999): Silentium! Reinbek bei Hamburg.

Hein Freund = Hein, Christoph (1987): Der fremde Freund. Novelle. 5. Aufl. Berlin/Weimar.

[Erstausg. Berlin/Weimar 1982].

Heinrich Soweit = Heinrich, Finn-Ole (2005): Soweit kein ungewohntes Wort. In: Heinrich,

Finn-Ole: Die Taschen voll Wasser. Erzählungen. Hamburg, S. 67-68.

Hildebrandt achtzig = Hildebrandt, Dieter (2007): Nie wieder achtzig! München .

Kirchhoff Widerfahrnis = Kirchhoff, Bodo (2016): Widerfahrnis. Eine Novelle. 4. Aufl.

Frankfurt a. M.

Lenz Deutschstunde = Lenz, Siegfried (1973): Deutschstunde. München.

Lenz Landesbühne = Lenz, Siegfried (2009): Landesbühne. Hamburg.

Lenz Überläufer = Lenz, Siegfried (2016): Der Überläufer. Roman. 7. Aufl. Hamburg.

Strittmatter Bienkopp = Strittmatter, Erwin (2003): Ole Bienkopp. Roman. (= Die DDR-Bibliothek 23).

Leipzig. [Erstausg. Berlin 1963].

Timm Sommer = Timm, Uwe (1977): Heißer Sommer. Reinbek bei Hamburg.

Werfel Blassblau = Werfel, Franz (2017): Eine blassblaue Frauenschrift. Köln. [Erstausg. Buenos

Aires 1941]. 


\section{Wissenschaftliche Literatur}

Adam, Jean-Michel (2005): La linguistique textuelle. Introduction à l'analyse textuelle des discours. Paris.

Ágel, Vilmos (2000): Valenztheorie. Tübingen.

Ágel, Vilmos (2015a): Grammatik und Literatur. Grammatische Eigentlichkeit bei Kehlmann, Timm, Liebmann, Handke, Strittmatter und Ruge. In: Brinker-von der Heyde, Claudia et al. (Hg.): Eigentlichkeit. Zum Verhältnis von Sprache, Sprechern und Welt. Berlin/München/ Boston, S. 159-174.

Ágel, Vilmos (2015b): Brisante Gegenstände. Zur valenztheoretischen Integrierbarkeit von Konstruktionen. In: Engelberg et al. (Hg.), S. 61-87.

Ágel, Vilmos/Kehrein, Roland (2013): Sogenannte Koordinationsellipsen. Von der Prosodie zur Theorie. In: Hennig, Mathilde (Hg.): Die Ellipse. Neue Perspektiven auf ein altes Phänomen. (= Linguistik - Impulse \& Tendenzen 52). Berlin/Boston, S. 107-158.

Allwood, Jens/Andersson, Lars-Gunnar/Dahl, Östen (1973): Logik für Linguisten. (= Romanistische Arbeitshefte 8). Tübingen.

Bassola, Péter/Schwinn, Horst (2016): Markierte Vorfeldbesetzungen im Deutschen. In: Dalmas, Martine/Fabricius-Hansen, Cathrine/Schwinn, Horst (Hg.): Variation im europäischen Kontrast. Untersuchungen zum Satzanfang im Deutschen, Französischen, Norwegischen, Polnischen und Ungarischen. (= Konvergenz und Divergenz 5). Berlin/ Boston, S. 229-259.

Christ, Rüdiger (2017): Niemand braucht das Prädikat. Zur Systematisierung der schulischen Satzgliedanalyse. In: Linguistische Berichte 250, S. 169-218.

Coseriu, Eugenio (1974): Synchronie, Diachronie und Geschichte. Das Problem des Sprachwandels. (= Internationale Bibliothek für Allgemeine Linguistik 3). München.

Coseriu, Eugenio (1987): Semantik und Grammatik. In: Coseriu, Eugenio (Hg.): Formen und Funktionen. Studien zur Grammatik. (= Konzepte der Sprach- und Literaturwissenschaft 33). Tübingen, S. 85-95. [Originalausg.: Moser, Hugo (Hg.) (1972): Neue Grammatiktheorien und ihre Anwendung auf das heutige Deutsch. (= Jahrbuch 1971 des Instituts für Deutsche Sprache). Düsseldorf].

Coseriu, Eugenio (1994): Textlinguistik. Eine Einführung. 3., überarb. u. erw. Aufl. Tübingen/ Basel.

Eisenberg, Peter (2006): Grundriss der deutschen Grammatik. Bd. 2: Der Satz. 3., durchges. Aufl. Stuttgart/Weimar.

Engelberg, Stefan et al. (Hg.) (2015): Argumentstruktur zwischen Valenz und Konstruktion. (= Studien zur Deutschen Sprache 68). Tübingen.

Fischer, Klaus (2003): Verb, Aussage, Valenzdefinition und Valenzrealisierung: Auf dem Weg zu einer typologisch adäquaten Valenztheorie. In: Willems, Klaas/Coene, Ann/Van Pottelberge, Jeroen (Hg.): Valenztheorie. Neuere Perspektiven. (= Studia Germanica Gandensia 2003/2). Gent, S. 14-64.

Fleischmann, Eberhard (1985): Kasustheorie und Translationslinguistik. Diss. Leipzig. GTA = Ágel, Vilmos (2017): Grammatische Textanalyse. Textglieder, Satzglieder, Wortgruppenglieder. Berlin/Boston.

Haspelmath, Martin (1993): More on the typology of inchoative/causative alternations. In: Comrie, Bernard/Polinsky, Maria (Hg.): Causatives and transitivity. (= Studies in Language Companion Series 23). Amsterdam/Philadelphia, S. 87-120. 
HdK = Pasch, Renate et al. (2003): Handbuch der deutschen Konnektoren. Linguistische Grundlagen der Beschreibung und syntaktische Merkmale der deutschen Satzverknüpfer (Konjunktionen, Satzadverbien und Partikeln). (= Schriften des Instituts für Deutsche Sprache 9). Berlin/New York.

Helbig, Gerhard (1992): Grammatiken und ihre Benutzer. In: Ágel, Vilmos/Hessky, Regina (Hg.): Offene Fragen - offene Antworten in der Sprachgermanistik. (= Reihe Germanistische Linguistik 128). Tübingen, S. 135-150.

Hennig, Mathilde/Buchwald-Wargenau, Isabel (2010): Ausdrucksarten - ein neuer Zugang zur Wortschatzvermittlung im DaF-Unterricht? In: Ferraresi, Gisela/Kresić, Marijana (Hg.): Neue Perspektiven auf das Verhältnis zwischen linguistischer und didaktischer Grammatik. Linguistik Online 41, S. 7-23.

Höllein, Dagobert (2019): Präpositionalobjekt vs. Adverbial. Die semantischen Rollen der Präpositionalobjekte. Berlin.

IDS-Grammatik (1997) = Zifonun, Gisela/Hoffmann, Ludger/Strecker, Bruno (1997a): Grammatik der deutschen Sprache. 3 Bde. (= Schriften des Instituts für Deutsche Sprache 7). Berlin/ New York.

Jacobs, Joachim (2008): Wozu Konstruktionen? In: Linguistische Berichte 213, S. 3-44. Jacobs, Joachim (2009): Valenzbindung oder Konstruktionsbindung? Eine Grundfrage der Grammatiktheorie. In: Zeitschrift für germanistische Linguistik 37, S. 490-513.

Knobloch, Clemens (1990): Wortarten und Satzglieder. Theoretische Überlegungen zu einem alten Problem. In: Beiträge zur Geschichte der deutschen Sprache und Literatur 112, S. $173-199$.

Schmidt, Jürgen Erich/Herrgen, Joachim (2011): Sprachdynamik. Eine Einführung in die moderne Regionalsprachenforschung. (= Grundlagen der Germanistik 49). Berlin.

Tesnière, Lucien (1976): Éléments de syntaxe structurale. 2., überarb. u. korr. Aufl. Paris. Tesnière, Lucien (1980): Grundzüge der strukturalen Syntax. Stuttgart.

Teuber, Oliver (2005): Analytische Verbformen im Deutschen. Syntax - Semantik - Grammatikalisierung. (= Germanistische Linguistik 18). Hildesheim/Zürich/New York.

Welke, Klaus (2005): Deutsche Syntax funktional. Perspektiviertheit syntaktischer Strukturen. 2., bearb. Aufl. (= Stauffenburg Linguistik 22). Tübingen.

Welke, Klaus (2011): Valenzgrammatik des Deutschen. Eine Einführung. Berlin/New York.

Welke, Klaus (2015): Wechselseitigkeit von Valenz und Konstruktion: Valenz als Grundvalenz. In: Engelberg et al. (Hg.), S. 35-59. 